\title{
Sensitive Dependence on Parameters of Continuous-time Nonlinear Dynamical Systems
}

\author{
E. S. Medeiros ${ }^{a}$,, I. L. Caldas ${ }^{a}$, M. S. Baptista ${ }^{b}$ \\ ${ }^{a}$ Institute of Physics, University of São Paulo, Rua do Matão, Travessa R 187, 05508-090, \\ São Paulo, Brazil \\ ${ }^{b}$ Institute for Complex Systems and Mathematical Biology, SUPA, University of Aberdeen, \\ AB24 3UE Aberdeen, United Kingdom
}

\begin{abstract}
The sensitive dependence of periodicity and chaos on parameters is investigated for three-dimensional nonlinear dynamical systems. Previous works have found that noninvertible low-dimensional maps present power-law exponents relating the uncertainty between periodicity and chaos to the precision on the system parameters. Furthermore, the values obtained for these exponents have been conjectured to be universal in these maps. However, confirmation of the observed exponent values in continuous-time systems remain an open question. In this work, we show that one of these exponents can also be found in different classes of three-dimensional continuous-time dynamical systems, suggesting that the sensitive dependence on parameters of deterministic nonlinear dynamical systems is typical.
\end{abstract}

Keywords: Fractal boundaries, Parameters Space, Complex Periodic windows PACS: 05.45.-a, 05.45.Df, 05.45.Pq

\section{Introduction}

The final asymptotic behavior of nonlinear dynamical systems can be severely affected by small perturbations in their control parameters [1]. The so-called parameter sensitivity has been experimentally observed in different areas of

Email address: esm@if.usp.br (E. S. Medeiros)

Preprint submitted to Journal of ${ }^{A} T_{E} X$ Templates

March 20, 2017 
${ }_{5}$ knowledge [2, 3, 4, 5]. The cause of this sensitivity is the existence of bifurcation sets in parameters spaces that, regardless of any scale, provoke several topological changes in the system dynamics. One of the most drastic example is the conversion of stable periodic into chaotic behavior, or vice-versa. The parameter sensitivity in this case dramatically limits the ability of someone surely set the parameters of a system to oscillate either in a chaotic or in a periodic behavior.

Following works studying the sensitivity of multiple attractors on the initial conditions and parameters [6, 7], the sensitive dependence of periodicity and chaos on parameters has been first addressed in 1985 by Grebogi et al. [8]. The authors have defined a scaling exponent, $\alpha$, such that for $\alpha$ lower than 1 , the system asymptotic solutions, periodic and chaotic, are regarded to be sensitive dependent on the system's parameters. By varying the unique parameter of an one-dimensional discrete-time system, the quadratic map, the authors have found the scaling exponent $\alpha$ to be equal to $0.413(5)$, indicating the parameter sensitivity in this system. Also, in 1985 [9], J.D. Farmer has proposed a different coarse-grained measure to quantify the parameter dependence of periodicity and chaos, and use it to confirm sensitive dependence on parameters in the quadratic and sine maps. In these works this exponent reflects the topology of the boundary between parameter regions leading to chaotic and periodic 25 behavior. On the other hand, Hunt et al. [10] have theoretically estimated the scaling exponent, $\alpha$, but only based on the topological properties of the boundaries between parameter regions describing large chaotic attractors (to be defined subsequently) and parameter regions describing periodic orbits of the quadratic map. In their analyses, they have found the scaling exponent 30 $\alpha$ to be equal to $0.51(3)$. This value of $\alpha$ obtained by Hunt et al. indicates a low parameter sensitivity when only large chaotic attractors are considered. This issue has been addressed in 2014 by Joglekar et al. [11, who found a general relationship between the two scaling exponent values previously defined. Moreover, they conjecture that these two values for the scaling exponent, $\alpha$, are universal for one-dimensional quadratic maps. 
However, a question that remains open at this point is whether these scaling exponents also apply to higher-dimensional systems, such the ones whose the dynamics is described by continuous-time equations, and more than one parameter is available to induce sensitivity.

In this work, we address the sensitive dependence on parameters of periodicity and chaos in two-dimensional parameters sets of continuous-time dynamical systems. Specifically, we quantify the sensitive on parameters of these systems by calculating the fraction of parameters that changes their corresponding asymptotic dynamical behavior under a given perturbation. We observe that such fraction of uncertain parameters scales as a power-law with the magnitude of the perturbation, and therefore, yields the scaling exponent, $\alpha$. Moreover, we verify that the scaling exponent value, measured considering any chaotic attractor, is roughly the same within the three different classes of continuoustime systems here investigated, agreeing with the value obtained for the onedimensional, sine, and quadratic maps. This result suggests that the scaling exponent $\alpha$ is a universal measure for the sensitive dependence of periodicity and chaos on parameters of nonlinear dynamical systems.

In parameters sets of nonlinear dynamical systems, the sensitive dependence of periodicity and chaos can be investigated by the way that their corresponding 55 parameter subsets are arranged. The parameters leading to chaos is interwoven with continuous sets of parameters leading to periodic stable behavior, called periodic windows. Periodic windows are parameter sets, which in this work is assumed to contain parameter sets corresponding to a periodic orbit and its eventual bifurcated harmonic solutions. On the other hand, we consider complex structures, i.e., regions of parameter sets that contain scale-free accumulations of periodic windows and also parameters corresponding to chaos. More specifically, as a system parameter is varied, the periodic trajectory with the lowest period, $p$, appearing for a parameter inside a periodic window, undergoes a cascade of period-doubling bifurcations resulting in a "small" $p$-band chaotic attractor. Subsequently, such chaotic attractor goes through an interior crisis, at which the complex structures, containing chaos and periodicity, ter- 
minates giving place to a large chaotic attractor that is larger than the $p$-band chaotic one (small chaotic attractor) [12, 11]. Furthermore, the chaotic regions describing attractors that become chaotic via period-doubling occurring inside a complex structure is also interwoven with other high-order complex structures that also contain parameters leading to chaos appearing via period-doubling cascades. The parameter region describing small chaotic attractors inside a lower-order complex structure becomes a region describing large chaotic attractors surrounding higher-order one. This hierarchical arrangement is repeated over and over again in all scales of parameter sets, generating the sensitive dependence on parameters in deterministic dynamical systems.

In Figure 1, we show a typical complex structure commonly observed in the two-dimensional parameter sets of several systems [13, 14, 15, 16, 17, 18, 19, 20, 21, 22. The white regions are periodic windows and represent parameters leadso ing to periodic solutions, gray represents the parameters leading to the small $p$-band chaotic attractors, and black represents the parameters leading to large chaotic attractors. Thus, in Figure 1, one can see a complex structure that is formed by the white and gray color while the regions outside the complex structure is black. Since this complex structure has the terminator boundary curve at a border collision crisis (upper part), it is a primary complex structure. The inset box whose amplification can be seen in the upper right corner shows other periodic windows laying on a region dominated by multi-band chaotic attractors, the gray region. Each one of these cascading higher-order periodic windows forms a higher-order complex structure, if its neighboring multi-band chaotic attractors are taken into consideration. Our analysis to measure the scaling exponent will be made considering such regions, where cascades of periodic windows accumulates into a primary complex structure.

In the last 20 years, the complex structures shown in Figure 1 have been numerically observed in parameter sets of a large numbers of systems ranging 95 from discrete-time biological oscillator to continuous-time lasers and chemical reactions models 13, 14, 15, 16, 17, 18, 19, 20, 21, 22. Furthermore, more recently, such periodic arrangements are being observed in parameter sets of 
electronic circuits in lab experiments [23, 24, 25, 26, 27, increasing the interest in the sensitive dependence on parameters.

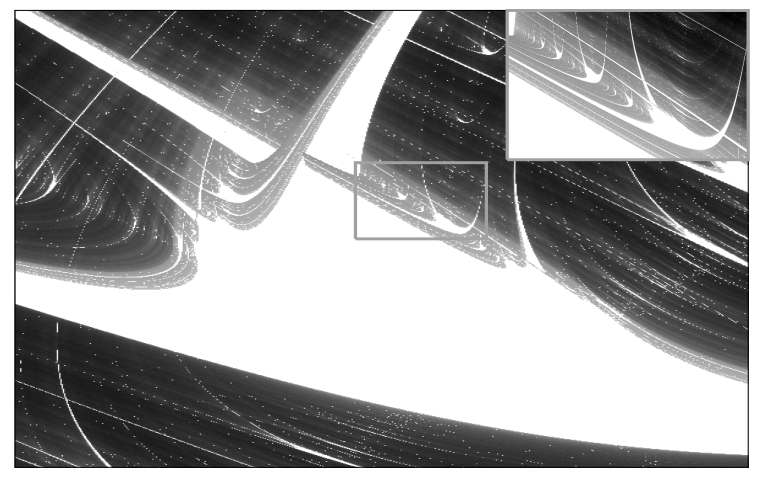

Figure 1: Typical two-dimensional $a \times b$ parameter set of nonlinear dynamical systems. The white color indicates the parameters leading to periodicity (periodic windows). The gray color indicates parameters corresponding to chaos via period-doubling bifurcation, and black color indicates parameters corresponding to chaos outside the windows (large chaotic attractors). The magnification shows a sequence of periodic windows immersed in a parameter region of chaos via period-doubling.

\section{Results}

Our numerical results are based on simulations of three different classes of continuous-time dynamical systems. In our calculations, we address parameter sets composed by sequences of high-order complex structure [28], as in the inset of Fig. 1. sequences occur for parameters inside a complex structure in the border with the large chaotic attractors located outside the complex structure. In our computations, we consider chaotic attractors of all sizes, and we did not consider systems for which the parameters spaces are Riddled or Wada as discussed in Refs. [29, 30].

To represent the class of oscillators for which the Shilnikov theorem can be applied [31, 32, 33, 34, 35, 36], we consider the Rössler oscillator. This system is described by the following set of nonlinear differential equations:

$$
\dot{x}=-y-z,
$$




$$
\begin{aligned}
\dot{y} & =x+a y, \\
\dot{z} & =(b+z) x-c z .
\end{aligned}
$$

and we study the parameter plane $a \times c$. The other parameter $b$ of Eq. (1) is fixed at $b=0.3$ [17.

The class of nonlinear forced oscillators are represented by the Morse oscillator which is governed by the following nonlinear differential equation [37]:

$$
\ddot{x}+d \dot{x}+8 e^{-x}\left(1-e^{-x}\right)=2.5 \cos (\omega t)
$$

and we study the parameter plane $\omega \times d$.

We also work with a loss-modulated $\mathrm{CO}_{2}$ laser described by a rate-equation with a time-dependent parameter:

$$
\begin{aligned}
& \dot{u}=\frac{1}{\tau}(z-k(t)) u, \\
& \dot{z}=\left(z_{0}-z\right) \gamma-u z,
\end{aligned}
$$

where $k(t)=k_{0}(1+a \cos 2 \pi f t)$. We study the $a \times f$ parameter plane. All other parameters are fixed: $\tau=3.5 \times 10^{9} s, \gamma=1.978 \times 10^{5} s^{-1}, z_{0}=0.175$, and $k_{0}=0.1731[15]$.

We numerically integrate Eq. from () to () using a Fourth-order Runge Kutta method and obtainthe two-dimensional parameter spaces of those systems. In those parameters sets, we select $N=3.0 \times 10^{4}$ pairs of random parameters, say $\left(a_{0}, b_{0}\right)$, uniformly space distributed and compute the largest Lyapunov exponents of the trajectory of the considered systems for these random parameters to determine if the correspondent state is periodic $(\lambda<0)$ or chaotic $(\lambda>0)$. To evaluate the parameter sensitivity is enough to consider only one direction in the parameter space. Hence, each pair of parameters is perturbed by an error $\varepsilon$ along one parameter, which we regard as the horizontal direction. This process generates $6.0 \times 10^{4}$ pairs $\left(a_{0} \pm \varepsilon, b_{0}\right)$ of parameters. We also obtain the 125 Lyapunov exponent of states corresponding to the perturbed parameter pairs. We then compare the parameters to their two correspondent perturbed pairs along the horizontal direction. If at least one of them has changed the final 
behavior (chaotic to periodic, or vice-versa), the pair $\left(a_{0}, b_{0}\right)$ is counted as an uncertain pair for the error value $\varepsilon$. If $u(\varepsilon)$ denotes the number of uncertain parameter pairs as a function of $\varepsilon$ for a selected region in the parameter set, then the fraction of uncertain parameters is calculated by $f(\varepsilon)=u(\varepsilon) / N$.

In Figure 2(Left), we show the parameter spaces for the three systems considered, for which $f(\varepsilon)$ is calculated. The black regions indicate the set of parameters leading to chaos, while the white regions correspond to parameters leading to periodic stable behavior. In these figures, complex structures, and periodic windows, are aligned along sequences accumulating in periodic regions of parameter sets. In Figure 2(Right), for the correspondent parameter space shown in Figure 2(Left), we show the fraction $f(\varepsilon)$ of uncertain periodic parameters as a function of the error $\varepsilon$. The straight line is a power-law fitting between $f(\varepsilon)$ and $\varepsilon$ which provides the uncertainty exponent $\alpha$.

We observe in Figure 2 that the exponent $\alpha$ is in the same confidence interval given by $\alpha=0.40(4)$ for the different classes of dynamical systems considered here. The standard deviation of $\alpha$ has been obtained by considering that the occurrence of uncertain parameters are random events. The results are shown in Table 1. The numerical value found for the exponent $\alpha$ relates the fraction of uncertain parameters to the accuracy in measuring them. For example, for the parameter set of the Rössler oscillator and with $\epsilon=0.005$ approximately $81 \%$ of the parameters are uncertain. If one increases the accuracy by factor 10, i.e., for $\varepsilon=0.0005$, approximately $32 \%$ of the parameters are still uncertain. The implication is that a high accuracy on parameters may be necessary to predict reliably the chaotic or periodic asymptotic behavior of a chaotic oscillator.

\begin{tabular}{|l|l|}
\hline Dynamical System & $\alpha$ \\
\hline \hline Rössler Oscillator & $0.41 \pm 0.04$ \\
\hline Morse Oscillator & $0.40 \pm 0.04$ \\
\hline $\mathrm{CO}_{2}$ Laser & $0.40 \pm 0.04$ \\
\hline
\end{tabular}

Table 1: In this table, for all dynamical systems investigated, we show in the first column the values of $\alpha$ obtained from the fitting shown in Figure 2(Right). 

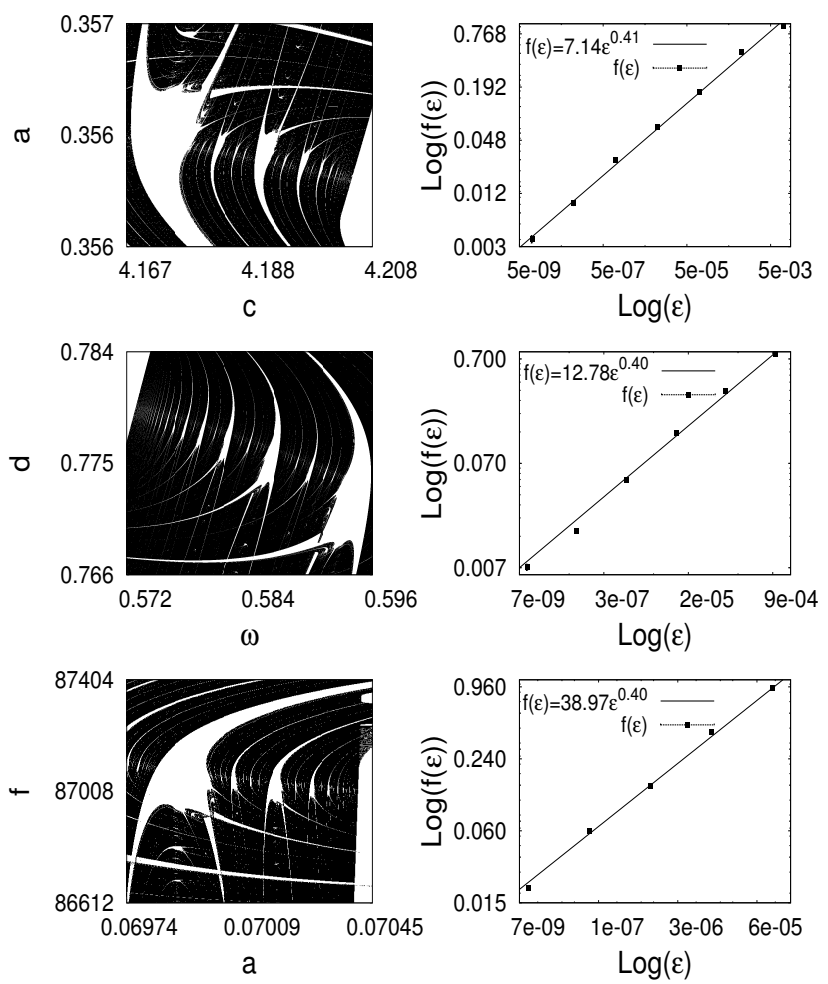

Figure 2: (Left) Two-dimensional parameter spaces of the three considered dynamical systems. Black regions represent the chaotic parameter set. White regions represent the periodic parameter set. (Right) The uncertain fraction $f(\varepsilon)$ of the chaotic sets shown in (Left) scales as power-law with the error $\varepsilon$. 


\section{Discussion}

In conclusion, we have verified that the scaling exponent, $\alpha$, applies for high-dimensional continuous-time systems, yielding a reliable measure for the

Moreover, our estimative for the scaling exponent agrees to the values argued to be universal in one-dimensional quadratic map. Therefore, our findings extend the universality of the scaling exponent $\alpha$ to a larger class of nonlinear dynamical system.

to predict reliably the chaotic or periodic asymptotic behavior of a chaotic oscillator.

\section{References}

\section{References}

[1] Y. A. Kuznetsov, Elements of applied bifurcation theory, Springer, United States of America, 2004.

[2] R. F. Costantino, R. A. Desharnais, J. M. Cushing, B. Dennis, Chaotic dynamics in an insect population, Science 275 (1997) 389.

[3] W. Ren, S. J. Hu, B. J. Zhang, F. Z. Wang, Y. F. Gong, J. X. Xu, Periodadding bifurcation with chaos in the interspike intervals generated by an experimental neural pacemaker, Int. J. of Bif. and Chaos 7 (1997) 1867.

[4] S. Valling, B. Krauskopf, T. Fordell, A. M. Lindberg, Experimental bifurcation diagram of a solid state laser with optical injection, Opt. Commun. $271(2007) 532$.

175

[5] Y. Kolokolov, A. Monovskaya, Estimating the uncertainty of the behavior of a pwm power converter by analyzing a set of experimental bifurcations diagrams, Int. J. Bif. and Chaos 23 (2013) 1350063. 
[6] C. Grebogi, E. Ott, J. A. Yorke, Crises, sudden changes in chaotic attractors, and transient chaos, Physica D 7 (1983) 181.

[7] S. W. McDonald, C. Grebogi, E. Ott, J. A. Yorke, Fractal basin boundaries, Physica D 17 (1985) 125.

[8] C. Grebogi, S. W. McDonald, E. Ott, J. A. Yorke, Exterior dimension of fat fractals, Phys. Lett. A 110 (1985) 01.

[9] J. D. Farmer, Sensitive dependence on parameters in nonlinear dynamics, Phys. Rev. lett. 55 (1985) 351.

[10] B. Hunt, E. Ott, Structure in the parameter dependence of order and chaos for the quadratic map, J. Phys. A: Math. Gen. 30 (1997) 7067.

[11] M. Joglekar, E. Ott, J. A. Yorke, Scaling of chaos versus periodicity: How certain is it that an attractor is chaotic?, Phys. Rev. Lett. 113 (2014) 084101.

[12] J. A. Yorke, C. Grebogi, E. Ott, L. Tedeschini-Lalli, Scaling behavior of windows in dissipative dynamical systems, Phys. Rev. Lett. 54 (1985) 1095.

[13] L. Glass, M. R. Guevara, J. Belair, A. Shrier, Global bifurcations of a periodically forced biological oscillator, Phys. Rev. A 29 (1984) 1348.

[14] M. S. Baptista, I. Caldas, Phase-locking and bifurcations of the sinusoidally-driven double scroll circuit, Nonlinear Dynamics 17 (2) (1998) 119.

[15] C. Bonatto, J. C. Garreau, J. A. C. Gallas, Self-similararities in the frequency-amplitude space of a loss-modulated $\mathrm{co}_{2}$ laser, Phys. Rev. Lett. $95(2005) 143905$.

[16] J. Slipantschuk, E. Ullner, M. S. Baptista, M. Zeineddine, M. Thiel, Abundace os stable periodic behavior in a red grouse population model with delay: A consequence of homoclicity, Chaos 20 (2010) 045117. 
[17] J. A. C. Gallas, The structure of infinite periodic and chaotic hub cascades in phase diagrams of simple autonomous flows, Int. J. Bif. and Chaos 20 (2010) 197.

[18] D. F. M. Oliveira, E. D. Leonel, Parameter space for a dissipative fermiulam model, New Journal of Physics 13 (12) (2011) 123012.

[19] H. Podhaisky, W. Marszalek, Bifurcations and synchronization of singularly perturbed oscillators: an application case study, Nonlinear Dynamics 69 (3) (2012) 949.

[20] A. Hoff, D. T. da Silva, C. Manchein, H. A. Albuquerque, Bifurcation structures and transient chaos in a four-dimensional chua model, Phys. Lett. A 378 (3) (2014) 171.

[21] D. V. Savin, A. P. Kuznetsov, A. V. Savin, U. Feudel, Different types of critical behavior in conservatively coupled hénon maps, Phys. Rev. E 91 (2015) 062905.

[22] D. R. da Costa, C. P. Dettmann, E. D. Leonel, Transport and dynamical properties for a bouncing ball model with regular and stochastic perturbations, Commun. in Nonlinear Sc. and Num. Sim. 20 (3) (2015) 871.

[23] D. M. Maranhão, M. S. Baptista, J. C. Sartoreli, I. L. Caldas, Experimental observation of a complex periodic window, Phys. Rev. E 77 (2008) 037202.

[24] R. Stoop, P. Benner, Y. Uwate, Real-world existence and origins of the spiral organization of shrimp-shaped domains, Phys. Rev. Lett. 105 (2010) 074102 .

[25] E. R. Viana, R. M. Rubinger, H. A. Albuquerque, A. G. de Oliveira, G. M. Ribeiro, High-resolution parameter space of an experimental chaotic circuit, Chaos 20 (2010) 0231101.

[26] E. R. Viana, R. M. Rubinger, H. A. Albuquerque, F. O. Dias, A. G. 
Oliveira, G. M. Ribeiro, Periodicity detection on the parameter-space ofaforced chua's circuit, Nonlinear Dynamics 67 (1) (2011) 385.

[27] C. Cabeza, C. A. Briozzo, R. Garcia, J. G. Freire, A. C. Marti, J. A. Gallas, Periodicity hubs and wide spirals in a two-component autonomous electronic circuit, Chaos, Solitons and Fractals 52 (2013) 59.

[28] E. S. Medeiros, R. O. Medrano-T, I. L. Caldas, S. L. T. de Souza, Torsionadding and asymptotic winding number for periodic window sequences, Phys. Lett. A 377 (2013) 628.

[29] Y.-C. Lai, R. L. Winslow, Riddled parameter space in spatiotemporal chaotic dynamical systems, Phys. Rev. Lett. 72 (1994) 1640.

[30] Y. Zhang, G. Luo, Unpredictability of the wada property in the parameter plane, Phys. Lett. A 376 (2012) 3060.

[31] P. Gaspard, R. Kapral, G. Nicolis, Bifurcation phenomena near homoclinic systems: A two-parameter analysis, J. Stat. Phys. 35 (1984) 697.

[32] R. O. Medrano-T, I. L. Caldas, Periodic windows distribution resulting from homoclinic bifurcations in the two-parameter space, ArXiv eprints arXiv: 1012.2241.

[33] R. Barrio, F. Blesa, S. Serrano, A. Shilnikov, Global organization of spiral structures in biparameter space of dissipative systems with shilnikov saddlefoci, Phys. Rev. E 84 (2011) 035201(R).

[34] R. Vitolo, P. Glendinning, J. A. C. Gallas, Global structure of periodicity hubs in lyapunov phase diagrams of dissipative flows, Phys. Rev. E 84 (2011) 016216.

[35] H. A. Albuquerque, P. C. Rech, Spiral periodic structure inside chaotic region in parameter-space of a chua circuit, Int. J. Circ. Theor. Appl. 40 (2012) 189. 
[36] R. Barrio, F. Blesa, S. Serrano, Topological changes in periodicity hubs of dissipative systems, Phys. Rev. Lett. 108 (2012) 214102.

[37] C. Scheffczyk, U. Parlitz, T. Kurz, W. Konp, W. Lauterborn, Comparison of bifurcation structures of driven dissipative nonlinear oscillators, Phys. Rev. A 43 (1991) 6495. 